\title{
Freie Leute? Freizügigkeit von Ehemaligen Leibeigenen in Österreich 1781-1848 (am Beispiel der Liechtensteinischen Herrschaft)
}

\section{The People Free? The Freedom of Movement of Former Serfs in Austria 1781-1848 (the Example of the Liechtenstein Domains)}

\author{
Zdeňka Stoklásková / zdenka.stoklaskova@email.cz \\ Historický ústav, Filozofická fakulta, Masarykova univerzita, Brno, CZ
}

\begin{abstract}
This paper attempts to answer the question concerning the degree to which the position of serfs changed after the abolition of serfdom in Austria. Were the people in fact free as has been stated time and again in the history books and textbooks? Research into two Liechtenstein estates - the South Moravian domain of Břeclav/Lundenburg and the Lower Austrian estate of Wilfersdorf was used to analyse the factors behind personal freedom of movement after the abolition of serfdom. Could former serfs travel freely to other estates or abroad? This narrow study showed that this was not the case until the end of the pre-March period, i.e. after the end of patrimonial administration. These findings go against traditional interpretations. The abolition of serfdom was undoubtedly a step towards a modern society, but its implementation did not result in the end of control mechanisms, as they were replaced by others, and not only in the developing legal system. A lesser-known new factor from the period was the restriction of demesnial jurisdiction, which was placed under the control of the regional authorities. However, the period of reform did usher in a new phenomenon: the legal right of former serfs to protection against seignorial powers.
\end{abstract}

\section{Keywords}

Princely House Liechtenstein, history, Moravia, lower Austria, abolition of serfdom

Diese Publikation entstand als Teil des Projekts des Exzellenz-Zentrums für interdisziplinäre Forschung in Kulturphänomenen der mitteleuropäischen Geschichte: Bild, Kommunikation, Verhalten (Tschechische Wissenschaftsstiftung Nr. 14-36521G). Übersetzt von der Autorin. 


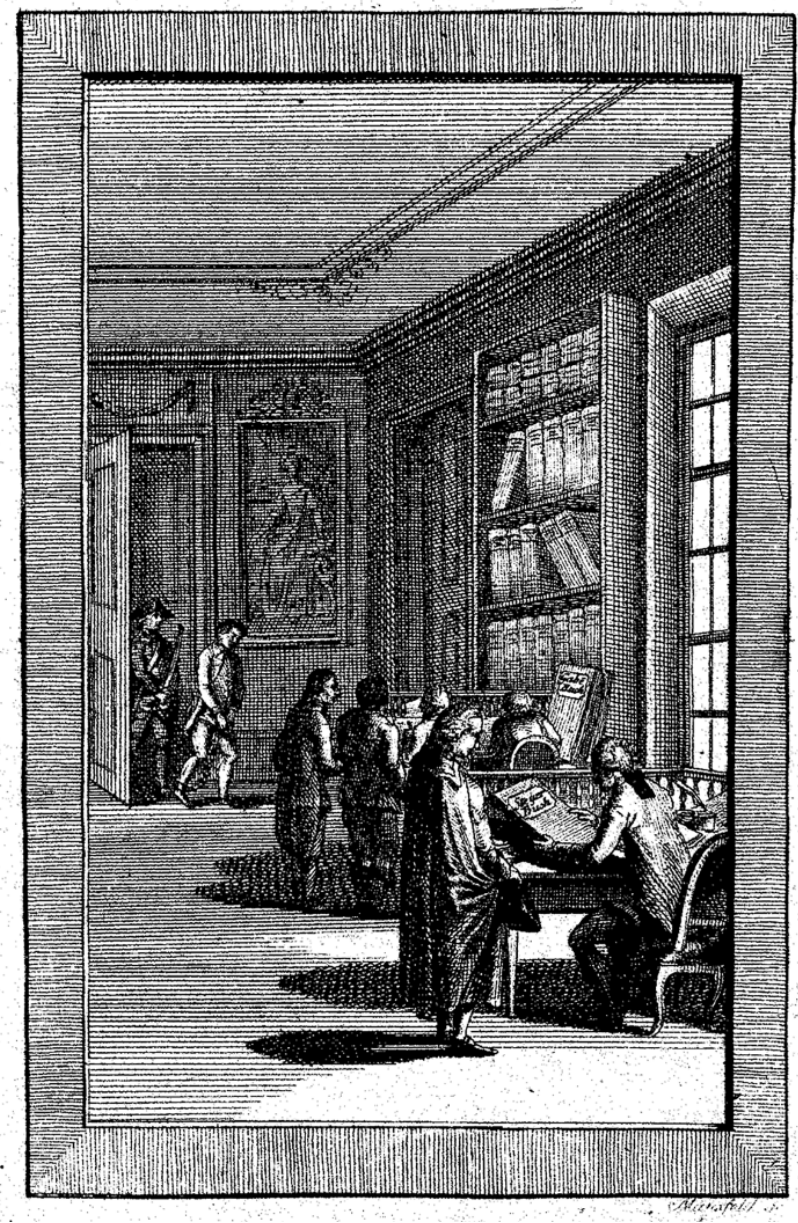

Abb. 1. Dominien. Kupferstich in Oktavformat. Joseph II. Gesetze, Bd. 8 (1787), Titelblatt, nicht paginiert. ${ }^{1}$

Das Titelblatt des achten Bandes der Josephinischen Gesetzsammlung versinnbildlicht grob Sinn und Zweck von Patenten und Verordnungen und damit auch die Quelle des "Gemeinwohls“ - nämlich die Arbeit von Untertanen. Die Abbildung zeigt eine Herrschaftskanzlei, deren halb geöffnete Tür die ganze Reihe von Untertanen ahnen lässt, die ergeben ihre Gelder mitbringen und ihre Pflichten bestätigen lassen: zwei Schreiber führen die wichtigsten Bücher des Dominiums: nämlich das Steuerbuch und das „Gabebuch“, also das Buch der Untertanen-Pflichten und -Abgaben. Beide Bücher sind symbolisch dargestellt, da sie in so einer Form nie bestanden hatten, der Zweck ist hier

$1 \quad$ Handbuch aller unter der Regierung des Kaisers Joseph II. für die K. K. Erbländer ergangenen Verordnungen und Gesetze in einer sistematischen Verbidnung (weiter siehe Joseph II. - Gesetze), 18 Bde., Wien 1785-1790, hier Bd. 1 (1785). Die Bezeichnung der Rechtsbilder stammt lediglich von der Verfasserin des Beitrags, die einzelnen Bilder sind im Original nicht betitelt. 
die symbolische Visualisierung der Wirtschaft im Dominium. In der Tür bringt eben ein Wachmann (oder ein Militärangehöriger) einen ungehorsamen Untertanen - und zwar in Fesseln. Das einfache Bild könnte als Allegorie (und eine kritische Vereinfachung) des herrschaftlichen Systems verstanden werden.

\section{Prolog:}

„(...) Folglich Seiner Maiestät oblige, eifrigst zu sorgen, damit Ihrer getreuen Länder und gehorsamsten Untertanen Wohlstand und gute Regierung befördert zu erhalten, und dann die Gott gefällige reine Justiz auf das genaueste gehandhabt werde.“2

Die Aufhebung der Leibeigenschaft und „die Einführung einer gemäßigten Untertänigkeit“ gehört unbestritten zu den wichtigsten Schritten des Josephinismus. Eine der drei neu verkündeten Freiheiten war die Möglichkeit, seine bisherige Herrschaft zu verlassen und sich bei einer neuen niederzulassen (und eventuell auszuwandern). Diese bekannte Tatsache steht in allen Lehrbüchern und Kompendien, die diese Zeit zum Thema haben.

\section{Freizügigkeit und Militär}

War die Freizügigkeit von Untertanen wirklich erreicht? Die Leibeigenschaftsaufhebung wurde gerade in der Zeit erlassen, als das stehende Heer eingeführt worden war. 1780 wurden die Konskriptionswerbbezirke geschaffen, die das Skelett der Organisation der Wehrpflicht wurden. Zehn Jahr zuvor war zum ersten Mal in der Geschichte des Vielvölkerstaates die männliche Population gezählt worden, um grundlegende, für die Existenz des stehenden Heeres unentbehrliche Fakten zu gewinnen. Bei der sog. Konskription im Jahre 1770 waren örtliche Würdenträger und Repräsentanten des Militärs anwesend, konskriptionspflichtige Männer mussten persönlich vor der Kommission erscheinen, da ihre Tauglichkeit beurteilt wurde. Für die Weiterexistenz des Militärs war es undenkbar, keine Übersicht über den aktuellen Stand der wehrpflichtigen Männer und „des militärischen Nachwuchses" zu haben.

Wichtig war nicht nur, wo ein wehrpflichtiger Mann politisch domiziliert war, (dies war eher für die eventuellen sozialen Bedürfnisse notwendig, jedoch für das Militär ziemlich unwichtig), sondern auch wie diese Person wieder zu finden und einzuziehen wäre. Diese Tendenz stand jedoch im Widerspruch zu der freien beruflichen Mobilität, die für den Staat unentbehrlich war. Wie der Mechanismus des (Wieder-)Findens eines zum Militärdienst eventuell tauglichen Mannes (in der etwas späteren Zeit, jedoch noch bei der Existenz der Untertänigkeit) praktisch verlief, beschrieb in seinen Erinnerungen ein böhmischer Wagnergeselle namens František Kebrdle, der sich gerade auf der Walz in der Steiermark befand. „Anfang März [1845] habe ich von zu Hause die Nachricht

2 Regierungsantritt. Joseph II. - Gesetze, Band 1 (1785), S. 12. 
erhalten, dass ich mich zur Musterung im Monat Mai stellen soll. Ich war durch diese Nachricht sehr betrübt, aber es musste so geschehen. So habe ich mich entschieden, dass ich an Ostern zu Hause sein werde. Ich bereitete mich also vor und nach dem Totensonntag, also vierzehn Tage vor den Feiertagen, machte ich mich am Montag auf den Weg. (...) Die Qual, was ich leiden musste, die ganze Zeit schneite es, besonders zwischen Budweis und Pisek waren Verwehungen, Schuhe ganz nass (...). Als ich nach Hause kam, war niemand in der Stube. Gerade danach kam die Mutter, sie konnte mich nicht erkennen, ich war vom Frost gebräunt und das Gesicht geschwollen (... $)^{{ }^{*}}$

Mit der Aufhebung der Leibeigenschaft wurde also nicht die volle Freizügigkeit von Untertanen erreicht, sondern es kam zur Überlagerung der Evidenz der Mobilität auf andere (staatliche) Subjekte. Es wurde jedoch, und zwar bis zur Aufhebung des Patrimonialsystems, die Dichotomie der politischen Macht belassen. Die Kompetenzen wurden zwar aufgeteilt, jedoch in bestimmten Bereichen kam es zum negativen Finanzkampf zwischen staatlichen und herrschaftlichen Strukturen. Die Evidenz der Bevölkerung war ein Bereich, wo es zu Überschneidungen kam.

Das neu eingeführte Konskriptionssystem, das zwar erstrangig zum Zweck des Militärs eingeführt wurde, zeigte sich so funktionsfähig, dass ihm sieben Jahre später, also im Jahre 1777, die Konskription der gesamten Bevölkerung folgte. Wer war der Träger der Konskription? Nicht mehr die Grundobrigkeit, sondern der Staat behufs der eigens geschaffenen Einheiten, der Konskriptionsbezirken, die mit Armenbezirken und Pfarreien oft identisch waren. Dies war in mehrerer Hinsicht sehr praktisch: falls sich Gemeinden auf dem Gebiet von zwei oder drei Herrschaften befanden, außerdem wurde wieder die katholische Kirche, der gerade das Armenwesen zum Teil abgenommen wurde, wieder in die Armenpflege (jetzt aber im Diensten des Staates) einbezogen. Der wichtige (wie auch typisch für den Josephinismus) Aspekt war auch das Sparen der staatlichen Finanzmittel, da ein Pfarrer meist nebst dem Schullehrer der gut einzige lese- und schreibkundige Mann in der Gemeinde war und die Tätigkeit für die Konskription unentgeltlich leistete.

Die Dichotomie der Kompetenzen blieb jedoch in der Freizügigkeitsevidenz, da Grundobrigkeiten Entlaßscheine für umziehende oder auswandernde Untertanen weiterhin ausstellten. Allein die Tatsache der Existenz von Entlaßscheinen zeigt Relikte der Leibeigenschaft, da keine wirkliche Freizügigkeit erreicht wurde, sondern eher „eine gemäßigte Untertänigkeit“. Entlaßscheine wurden nicht nur zum wichtigsten Kontrollmechanismus der Mobilität, sondern waren auch eminent wichtig in wirtschaftlicher Hinsicht (Robotentlastung), die hier jedoch nicht zu Thema ist.

\section{Der Mechanismus der Austellung von Entlaßscheinen}

Für die praktische Realisierung der Ausstellung von Entlaßscheinen wurden Sonden in Archivmaterial der Liechtensteinischen Herrschaftenen in Mähren (Herrschaft Lunden-

3 Kebrdle, František: Paměti [Erinnerungen]. Archiv Národního technického muzea v Praze [Archiv des Nationalen technischen Museums in Prag], Sbírka vzpomínek a rukopisů [Sammlung von Erinnerungen und Handschriften], Nr. 1609, S. 14-16. 
burg/Břeclav) und Niederösterreich (Herrschaft Wilfersdorf) gemacht. ${ }^{4}$ Das Ziel war, die Existenz der Entlaßscheine auch nach der Aufhebung der Leibeigenschaft zu belegen und der Mechanismus der Entlassung festzustellen. Die grundlegende Frage war, ob die Entlaßscheine auch wirklich bis zur Aufhebung des Patrimonialsystems bestanden hatten oder ob eventuell die Freizügigkeit allmählich offiziell ohne die Zustimmung der Herrschaft geschah, d. h. ob wirklich bis zum Jahre 1848 die Praxis der Kontrolle der Freizügigkeit vollgezogen wurde. Es wurden deswegen chronologische Sonden durchgeführt, und zwar bis zum Jahre 1848 .

Mobilität von Untertanen in der Liechtensteinischen Herrschaft Lundenburg 18411849. Analyse von Entlaßgesuchen. MLA, F 43, Karton 413, Registratur-Materie 9, Fascikel 1-9.

Entlassungen von Untertanen der Liechtensteinischen Herrschaft Lundenburg in Gerichtsbarkeiten anderer konskribierten Länder

\begin{tabular}{|r|r|}
\hline 1841 & 21 \\
\hline 1842 & 33 \\
\hline 1843 & 31 \\
\hline 1844 & 32 \\
\hline 1845 & 42 \\
\hline 1846 & 50 \\
\hline 1847 & 31 \\
\hline 1848 & 31 \\
\hline 1849 & 39 \\
\hline Zusammen & 310 \\
\hline
\end{tabular}

Entlassungen fremdherrschaftlicher Untertanen in die Liechtensteinische Jurisdiktion Herrschaft Lundenburg

\begin{tabular}{|r|r|}
\hline 1841 & 56 \\
\hline 1842 & 56 \\
\hline 1843 & 51 \\
\hline 1844 & 25 \\
\hline 1845 & 43 \\
\hline 1846 & 42 \\
\hline 1847 & 62 \\
\hline 1848 & 61 \\
\hline 1849 & 38 \\
\hline Zusammen & 434 \\
\hline
\end{tabular}

In den vierziger Jahren des 19. Jahrhunderts gewann die Liechtensteinische Herrschaft Lundenburg um 124 Untertanen mehr, als sie aus ihrer Obrigkeit entließ. Die Interpretierung dieser Tatsache ist aber äußerst schwierig: heißt dies also, dass Untertanen in der Herrschaft Lundenburg bessere Bedingungen für das Alltagsleben hatten und

4 Für den Toponym Lundenburg, tschechisch Břeclav, wird weiterhin lediglich die deutsche Form verwendet. 
deswegen diese Herrschaft zum gesuchten Gebiet wurde? Die These, dass die Liechtenstein gute Herren waren und deswegen ihr Herrschaftsgebiet bei Untertanen als günstig angesehen wurde, ist noch problematischer. Ein Teil der in die Lundenburger Herrschaft Entlassenen blieben weiterhin Liechtensteinische Untertanen, aber von einer anderen Herrschaft, meist einer der mährischen oder der niederösterreichischen. Die Landesgrenze spielte in diesem Zusammenhang überhaupt keine Rolle.

Dies war der Fall von Anton Batka, der in Hohenau in Niederösterreich geboren wurde, aber Lundenburger Untertan war, wohin er sich mit seiner Mutter bereits „vor Jahren" begeben hatte; wahrscheinlich haben beide die Lundenburger Zuständigkeit angenommen oder hatten sie immer besessen und in Hohenau nur zeitweilig gelebt hatten. ${ }^{5}$ Dies ist ein weiterer Beleg, dass Ämter zwischen Geburtsort und Zuständigkeit genau unterschieden, obwohl dem immer noch gültigen Patent vom 1804 der Geburtsort auch der Zuständigkeitsort sein konnte, jedoch dies in der Praxis kaum realisiert wurde. ${ }^{6}$ Anton Batka kaufte in Kostel (Podivín) die Chaluppe Nr. 244, womit er den Anspruch nicht nur auf die Kostel Gerichtsbarkeit, sondern auch an das Bürgerrecht erwarb. Seiner durch den Magistrat Kostel realisierten Bitte um die Entlassung aus der Lundenburger Jurisdiktion wurde am 18. Februar 1847 stattgegeben. $^{7}$

Wozu ist also solch eine Statistik von entlassenen und aufgenommen Untertanen eigentlich gut? Erstens wurde damit die Existenz der Entlaßscheine bis zur Aufhebung des Patrimonialsystems belegt und damit eine der gestellten Fragen beantwortet wurde. Zweitens ermöglicht uns diese Statistik die detaillierte Einsicht nicht nur in die Mobilität von Untertanen, sondern auch in die sozialen Zusammenhänge der herrschaftlichen Macht. Zusammenfassend kann man nach der Interpretierung der Existenz von Entlaßscheinen fragen. Sind sie als Relikt des Feudalismus, bzw. der obrigkeitlichen Macht anzusehen? Bis zu einem gewissen Grad sicher ja, aber sie können als eine Überbrückung der „alten“ und der „neuen“ Zeit verstanden werden. Sie sind die Verkörperung der Übertragung der Verwaltung vom Staat an Herrschaften. Natürlich handelst es sich um Bereiche, die auch für die Herrschaftsverwaltung wichtig sind: Anzahl von Untertanen, deren soziale Stellung und davon der abgeleitete Anspruch auf die eventuelle Armenversorgung. Der ganze Prozess ist für den Staat eminent wichtig, aber sehr bequem, da er nicht die damit verbundenen Mittel tragen muss, noch schlimmer (für die Dominien), der Staat besitzt das Kontroll- und eventuell Sanktionsrecht, ohne jedoch eventuelle Kosten übernehmen zu müssen.

5 Die nicht weit voneinander liegenden erwähnten Ortschaften trennt heute die Staatsgrenze, jedoch im 19. Jahrhundert war dies die Landesgrenze, die im Rahmen des Liechtensteinischen Dominiums keine Rolle spielte.

6 Eine Analyse der Problematik der Zuständigkeit als Problem der Armenfürsorge vgl. Stoklásková, Zdeňka: Die Erfassung der Lohnarbeiter in der Stadt als Instrument der Armenfürsorge. In: „Chudé budete míti mezi sebou stále..." Od charity k sociální politice ve městech 18.-20. století. [„Arme werden sie unter sich stets haben ..." Von der Charitas zur Sozialpolitik in Städten im 18. bis zum 20. Jahrhundert]. Documenta Pragensia XXXIV, Praha 2015, S. 499-521.

7 Moravský zemský archiv v Brně, [Mährisches Landesarchiv in Brünn], (weiter siehe: MZA Brno), Herrschaft Lundenburg F 43, Karton 412, Fascikel Nr. 7, Registratur-Materie 8. 
Entlaßgesuche bieten eine einmalige Einsicht nicht nur in die Mobilität von Untertanen, sondern auch in andere Bereiche des alltäglichen Lebens auf dem Lande, die jedoch hier nicht Thema der Forschung sind: die wirtschaftliche und soziale Lage von Antragstellern, die häufige Minderjährigkeit bei Eheschließungen, der Umkreis der Familien- und Berufsmobilität usw. Im Zusammenhang mit dem Thema wurden besonders Zuständigkeitsaspekt und Grund der Entlassung verfolgt. Die Zuständigkeit war fast bei allen Antragstellern richtig. Es ist zu bewundern, dass in einer noch nicht alphabetisierten Gesellschaft die Feststellung der Zuständigkeit kaum ein Problem war. Die Mehrheit von Untertanen wusste genau ihr politisches Domizil, bzw. ihre Zuständigkeitsobrigkeit.

Eine Ausnahme war ein gewisser Joseph Kutschera, der am 29. Januar 1847 mittels des Herrschaftsamtes Feldsberg (damals Niederösterreich) um seinen Entlaßschein ansuchte. Der Grund der Entlassung war die bevorstehende Heirat mit einer gewissen Theresia Malinkowitsch aus Oberthemenau (Charvátská Nová Ves), ebenfalls Land unter den Enns. Die gebetene (mährische) Herrschaft Lundenburg lehnte es gleich am nächsten Tag ab, Joseph Kutschera zu entlassen mit dem Hinweis, dass dieser nicht ihr Jurisdizent sei und nur zufällig in Lundenburg geboren sei. Den Geburtsort hielt Josef Kutschera wahrscheinlich für sein politisches Domizil und gab ihn falsch bei der Feldsberger Herrschaft an. Die Herrschaft in Lundenburg hatte offensichtlich eine sehr gute Übersicht über ihre Jurisdizenten und eine richtige Kenntnis der Vorschriften, da sie nebenbei mitteilte, dass die Entlassung von Joseph Kutschera nicht stattfinden müsse, da der Antragsteller bereits „Jurisdicent Einer löblichen Herrschaft seyn dürfte“, da (nach seiner Angabe) seine Mutter in Themenau (Poštorná) einen Ansässigen geehelicht habe und dort auch gestorben sei. Die Entlassung war hier also überflüssig, da Joseph Kutschera bereits in die Herrschaft Feldsberg zuständig war (jedoch ohne davon selbst zu wissen). Das Einzige Überflüssige war wahrscheinlich die Summe von sechs Kreuzern, die für die Entlassung als Bearbeitungsgebühr taxiert wurde. ${ }^{8}$

Der Grund der meisten Gesuche um die Entlassung aus der Jurisdiktion war die bevorstehende Heirat, die Übernahme einer Bauernwirtschaft oder das Antreten eines Gewerbes. Der Mechanismus der Entlassung ging überraschender Weise nicht von der „alten“, sondern von der „neuen“ Obrigkeit aus, die sich an die „alte“ „dienstfreundlich“ wandte, die betreffende Person aus ihrer Jurisdiktion zu entlassen. Es wurde kein Fall gefunden, bei dem die Entlassung verweigert wurde, falls an der Zuständigkeit der Person keinen Zweifel bestand. Ganz im Gegenteil, bewundernswert ist die Geschwindigkeit, mit der die Gesuche erledigt wurden. In der Regel kam die Entlassung in drei Tagen zustande, jedoch war keine Ausnahme die Fertigstellung des Entlaßscheines in zwei Tagen oder bereits am nächsten Tag. Es war tatsächlich eine „dienstfreundliche“ Tat gegenüber der anderen Herrschaft und dem Untertan.

Der häufiste Grund zum Entlassen aus der Jurisdiktion war die bevorstehende Heirat. Es ließen sich keine Unterschiede in der Anzahl der Gesuche zwischen Männer und Frauen finden. Bei Verlobten israelitischer Religion zog in der Regel die Braut zum Bräutigam, denn die Heirat war logischerweise mit einer Familiantenstelle verknüpft

8 Ebenda. 
(in Mähren waren die Familiantenstellen an die Judengemeinde gebunden). So war dies bei Johanna, Tochter des Kosteler Familianten Aron Kohn, die nach der Intervention des Verwaltungsamtes in Göding (Hodonín) aus der Lundenburger Jurisdiktion entlassen wurde, um den Gödinger Familianten Emauel Körner heiraten zu können. Das Gesuch wurde am 30. Juli datiert, am 1. August 1845 wurde Johanna Kohn aus der Lundenburger Gerichtsbarkeit entlassen. ${ }^{9}$ Christliche Verlobte entschieden sich wahrscheinlich der wirtschaftlichen Lage nach, wo sie nach der Hochzeit leben wollten, bei der Braut oder beim Bräutigam. Sehr häufig war die Entlassung in eine andere, aber ebenso Liechtensteinische Herrschaft. So ein Fall war eine gewisse Katharina Hackala aus der Gemeinde Landshut (Lanžhot), die den Untertan der Herrschaft Pawlovitz (Velké Pavlovice) ehelichen wollte. Das am 20. Januar 1847 in Pawlowitz datierte Gesuch kam in Lundenburg am 23. Januar an, am 25. Januar 1847 wurde Katharina Hackala aus der Jurisdiktion entlassen.

Den zweitwichtigsten Grund für die Niederlassung auf einer anderen Herrschaft stellte besonders bei Männern die Ausübung eines Gewerbes dar, wie bei Anton Mikusch aus Bilowitz (Velké Bílovice) in der Herrschaft Lundenburg. Dem ledigen und volljährigen Anton Mikusch wurde im Markt Heinfeld die Büchsenmachergewerbeausübung verliehen. Das Gesuch der zuständigen (niederösterreichischen) Stiftsherrschaft Lilienfeld und Kreisbach kam samt der Aufnahmsurkunde am 25. April 1847 in Lundenburg an, am nächsten Tag wurde Anton Mikusch aus der Jurisdiktion entlassen. Man kann vermuten, dass er mit einer Verleihung der Gewerbeausübung auch das Bürgerrecht in Hainberg bekam. ${ }^{10}$

Manchmal bedeutet die Aufnahme in eine andere Jurisdiktion einen gesellschaftlichen Aufstieg, wie bei Wilhelm Haar. Der ledige, fünfundzwanzigjährige in Zlin geborene Tapeziergeselle, der eine ordentliche Wanderbewilligung seiner zuständigen Lundenburger Herrschaft besaß, etablierte sich in Wien so gut, dass ihm die Ausübung seines Gewerbes mit dem Bürgerrecht verliehen wurde. Noch am selben Tag, am 22. April 1847, wandte sich das Wiener Magistrat an die Lundenburger Herrschaft mit der Bitte um die „baldgefällige Einsendung“ der Entlaßurkunde. ${ }^{11}$ Für den Magistrat war auch der Standpunkt der zuständigen militärischen Subjekte wichtig, d. h. ob Wilhelm Haar nicht wehrpflichtig ist. Jedoch hatte nach der Verleihung des Bürgerrechtes auch der eventuell zum Militärdienst vorgemerkte Geselle eine sehr gute Aussicht auf die Befreiung, denn die Ausübung des Gewerbes stand an der ersten Stelle in der Hierarchie der Befreiung vom Militärdienst. Am 2. Mai 1847 wurde Wilhelm Haar aus der Lundenburger Jurisdiktion entlassen.

Wilhelm Haar bekam das Bürgerrecht, deswegen wurde er in die Wiener Jurisdiktion problemlos aufgenommen. Dies war bei nicht bemittelten Personen fast ausgeschlossen, da der Wiener Magistrat sich heftig gegen eventuell sozial bedürftige Personen wehrte. Die grundsätzliche Regelung der Änderung des politischen Domizils wurde zwar we-

9 MZA Brno, Herrschaft Břeclav (Lundenburg), F 43, Karton 412, Fasciklel Nr. 5, Registratur-Materie 8.

10 MZA Brno, Herrschaft Břeclav (Lundenburg), F 43, Karton 412, Fasciklel Nr. 7, Registratur-Materie 8.

11 Ebenda. 
sentlich später, erst 1849 publiziert, aber große Agglomerationen setzten alle möglichen gesetzlichen Bremsen, um nicht fremde unbemittelte Personen aufnehmen zu müssen. Wien als „Schmelztiegel“ aller Arbeitsuchenden war besonders überlaufen. Magistrate einiger großer Städte führten eine Evidenz der fremden Arbeitskräfte (Fabrikarbeiter und Dienstpersonal), die regelmäßig kontrolliert wurde. Falls deren legale Urkunde (Konskriptionsbescheinigung oder später Heimatschein) abgelaufen war, wurde der Träger zur Verlängerung bei seiner Heimatgemeinde aufgefordert, worauf er unentgeltlich Anspruch hatte. Seine Gemeinde durfte die Ausstellung eines Heimatscheines nicht verweigern, wenn über die Zuständigkeit kein Zweifel bestand. Dies war ein wichtiger Kontrollmechanismus der Magistrate, denn mit einem abgelaufenen Heimatschein bestand ein latenter Anspruch auf die stillschweigend ersessene Heimatzuständigkeit. Mit einer neuen oder verlängerten Urkunde einer Arbeitskraft war dann der Magistrat vor dem latenten Anspruch auf Armenversorgung "geschützt“.

Sehr behutsam ging der Wiener Magistrat bei der Erteilung von Ehebewilligungen vor, bei der auch häufig Entlaßscheine ausgestellt wurden. So war dies beim Heiratsgesuch von Andreas Marker, einem einundvierzigjährigen Packfongarbeiter aus Obersulz (Niederösterreich), der im Oktober 1845 in Wien um die Erteilung des politischen Ehekonsenses bat. Die Braut war die zweiundvierzigjährige Catharina Piruschka von Dalleschitz (Dalešice) in Mähren; die Brautleute, beide katholischer Religion und ledigen Standes, waren „wegen ausgewiesener Erwerbsfähigkeit zur Ehebewilligung geeignet“, bedurften jedoch der Meldezettel ihrer Zuständigkeitsbehörden. Um eventuell zu verhindern, dass die Obrigkeit des Bittstellers einen Entlaßschein ausstellt, machte der Wiener Magistrat aufmerksam, dass er „bloß in den Fällen des angesuchten Bürgerrechtes eines Hausbesitzers, einer Handlung oder eines Gewerbes um die förmliche Entlassung einzuschreiten pflegt, (hier dagegen) kein Grund vorliegt, um diese Entlassung einzuschreiten. "12 Der Wiener Magistratsbeamte Pelikan fügte am 14. Oktober 1845 noch bei, dass „diesem Schreiben ein legal gestandenes Gesuch zu Grund liege“, womit er den Packfongarbeiter vor den eventuellen Sanktionen seiner Obrigkeit und von der eventuellen Ausweisung aus der Stadt schützen wollte, um also die Legalität des Gesuches zu unterstreichen. Übrigens gab es für eine Ausweisung keinen Grund, da Andreas Marker mit einer gültigen Aufenthaltsbewilligung versehen war, deren Gültigkeit von einer (vermuteten) Urkunde seiner Herrschaft abhängig war. Der Prozess war ganz in Einvernehmen der gültigen Gesetze.

In der Sonde der untersuchten Entlassungen und Aufnahmen vor allem auf den Liechteinischen Herrschaften spielte das Geschlecht keine Rolle, d. h. es ließe sich kein Unterschied beobachten, ob die Frau zum Mann zog (und seine Jurisdiktion übernahm) oder der Mann zur Frau (mit dem Übertreten in ihre Gerichtsbarkeit). Theoretisch waren beide Möglichkeiten gleich, jedoch wurden sie bloß auf dem Lande praktiziert. Große Städte weigerten sich, unbemittelte Fremde aufzunehmen, sogar auch den Ehemann, wenn die Frau ihre Jurisdicentin war. Dies war auch der Fall von Franziska Lutzer, einer sechsunddreißigjährigen Handschuhmacherin aus Wien, die einen gewissen Franz

12 LIECHTENSTEIN. The Princely Collections. Hausarchiv Liechtenstein (HAL Wien). Hausarchiv der regierenden Fürsten von Liechtenstein in Wien (HAL Wien), Herrschaft Wilfersdorf, H 1837. 
Kwasinger, einen siebenundzwanzigjährigen Goldarbeitergesellen, heiraten wollte. Über die bevorstehende Heirat wissen wir aus dem Gesuch des Bräutigams an den Wiener Magistrat, der sich vorschriftsgemäß mit der Bitte um die Ausstellung des politischen Ehekonsenses an die Liechtensteinische Herrschaft Lundenburg wandte. Beide Brautleute waren ledig, katholischer Religion und, was erstrangig wichtig war, „wegen ausgewiesener Erwerbsfähigkeit zur Erlangung dieser Ehebewilligung geeignet“. Der Ehe stand also kein Hindernis im Weg, jedoch wurde auch überprüft, ob die Trauung wirklich erfolgte. Die gebürtige Wienerin Franziska war höchstwahrscheinlich nach der Heirat plötzlich in Wien eine fremde Person, da sie dem $§ 92$ des Allgemeinen Bürgerlichen Gesetzbuches als verheiratete Frau der Zuständigkeit ihres Gatten folgen musste. Im Falle von Verarmung, Krankheit, Tode des Ehegatten oder einem anderen schweren Schicksal wäre sie nicht nur mit der Bitte um Unterstützung abgewiesen, sondern auch aus der Stadt nach Lundenburg „nach Hause“ abgeschoben, wo sie niemand kannte, jedoch dem Gesetz nach dort zuständig war.

Selten kam es zum doppelten Grund der Entlassung aus der Gerichtsbarkeit: die Eheschließung und der Erwerb eines Hauses, wie bei dem ledigen und volljährigen Lundenburger "Inwohner“ Johann Mikosch (sein Vater besaß das Haus Nr. 52 in Lundenburg), der in die Jurisdiktion der niederösterreichischen Herrschaft Kreuzstetten aufgenommen wurde. Johann Mikosch kaufte das Haus Nr. 99 in Niederkreuzstetten mit der Absicht, sich dort niederzulassen und die minderjährige Katharina Leberwurst aus Wolkersdort zu heiraten. Die Herrschaft Kreuzstetten stellte ihm am 14. August 1843 eine Aufnahmsurkunde aus, nachdem er vorschriftlich die vorläufige Bewilligung Lundenburger Herrschaftskanzlei besorgte und dann den Entlaßschein seiner Herrschaft vorgelegt hatte. ${ }^{13}$

Die Minderjährigkeit der Braut war wirklich keine Ausnahme, eher gehörte sie zu den üblichen Tatsachen. Für die Ehe wurde Minderjährigkeit als kein Hindernis angesehen, es musste jedoch die schriftliche Zustimmung des Vormunds vorgelegt werden. So war es bei Ludmila Hollubetz, der minderjährigen Tochter nach Viktorin Hlawačz, Häusler in Mikultschitz (Mikulčice), die Kaspar Fiala, Häusler in Luschitz (Lužice) heiraten wollte. Das Verwaltungsamt in Göding (Hodonín), die Obrigkeit von Kaspar Fiala, bat nicht nur um die Entlassung von Ludmila Hollubetz, sondern auch um die vormundschaftliche Zustimmung. Der nicht des Schreibens kundige Vormund Augustin Rottasek gab zu Protokoll nicht nur seine Zustimmung, sondern eher eine Freude angesichts der bevorstehenden Heirat: „Indessen die Ludmila Hollubetz durch die Verehelichung mit dem Luschitzer Häusler Kaspar Fiala ihre Versorgung findt, da mir derselbe als ein ehrlicher Mann bekannt ist, so habe ich gegen die angesagte Entlassung zu ihrer Verehelichung nichts anzuwenden, sondern sie ist mir sehr wünschenswert." 14

Das Ziel des Beitrags war, den Mechanismus der Entlassung festzustellen. Dem erhaltenen Archivmaterial nach scheint der ganze Prozess der Entlassung und der Aufnahme folgende Phasen haben: zuerst die vorläufige Bewilligung der ursprünglichen

13 MLA Brno, Herrschaft Lundenburg F 43, Karton 412, Fascikel Nr. 3, Registratur-Materie 8.

14 MZA Brno, Herrschaft Břeclav (Lundenburg), F 43, Karton 412, Fascikel Nr. 2, Registratur-Materie 8. 


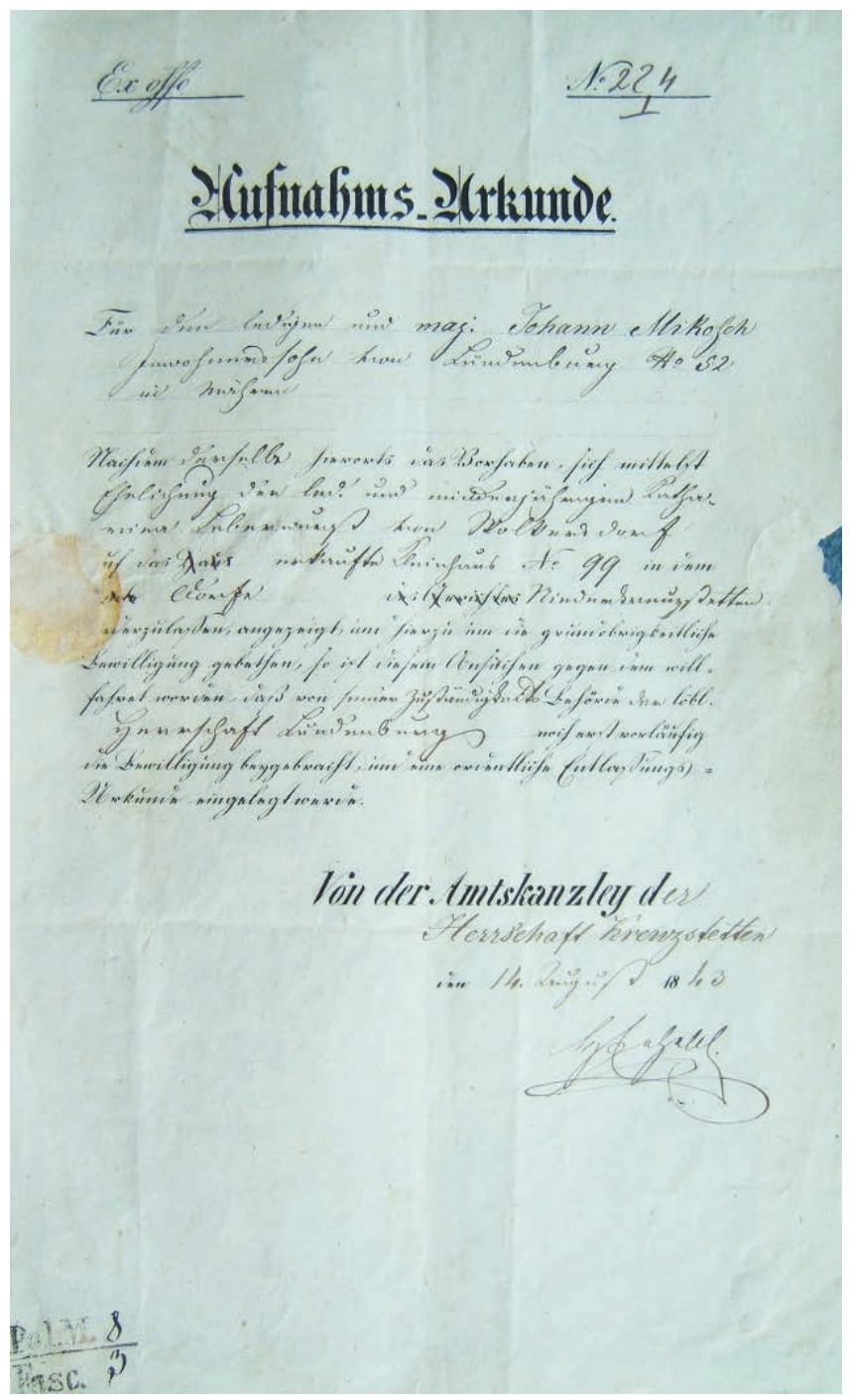

Abb. 2. Aufnahmsurkunde von Johann Mikosch, ausgestellt von Herrschaft Kreuzstetten am 14. August 1843. MZA Brno, F 43, Karton 412, F. 7.

Herrschaft, dann das Ansuchen der neuen Herrschaft, die Entlassung von der alten Herrschaft und die Aufnahme in die neue Jurisdiktion, die erst nach dem Vorlegen des Entlaßscheines zustande kam. Der beschriebene Weg ist bloß theoretisch, das es leider nicht gelang, bei einem Individuum den ganzen bürokratischen Weg zu finden; die Hypothese ist durch kollektive biographische Methode entstanden. ${ }^{15}$

15 Die Forschung war auch durch den Umstand erschwert, dass Archivmaterial von Liechtensteinischen Herrschaften in vier verschiedenen Archiven liegt, die ganz unterschiedlich manipuliert sind. 


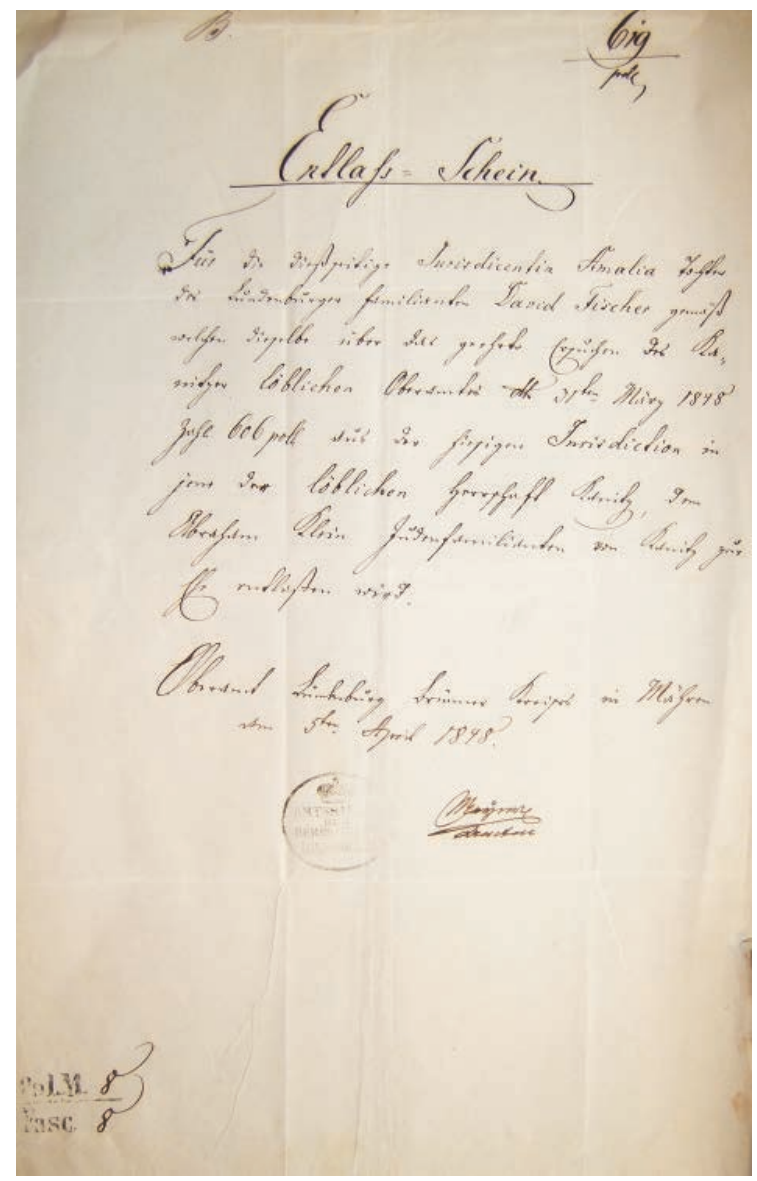

Abb. 3. Entlaßschein von Amalia Fischer, ausgestellt von Herrschaft Lundenburg am 5. April 1848. MZA, F 43, Karton 412, F. 8.

Eine theoretische Frage, die sich beim Lesen des Archivmaterials aufdrängte: Was passierte, wenn die Ursache, weswegen um Entlassung angesucht wurde, nicht zustande kam? Blieb der Mensch in der alten oder in der neuen Herrschaft? Hatte es für ihn bürokratische, soziale oder andere Konsequenzen? Dank des Falls einer gewissen Amalia Fischer konnte dies beantwortet werden. Das Oberamt in Kanitz (Dolní Kounice) bat am 31. März 1848 die Lundenburger Herrschaft um die Entlassung eben jener Amalia Fischer, Tochter von David Fischer aus Lundenburg. Abraham Klein, Uhrmacher und Judenfamiliant aus Kanitz erklärte am Oberamt in Kanitz seinen Willen, Amalia Fischer zu ehelichen und wandte sich deswegen an seine Zuständigkeitsobrigkeit. Das Gesuch kam am 4. April 1848 in Lundenburg an und gleich am nächsten Tag wurde Amalia Fischer aus der Lundenburger Jurisdiktion entlassen. Die Ehe kam aber aus unbekannten Gründen nicht zustande. Amalia Fischer kehrte in ihre Familie zurück und ihr Entlaßschein wurde tatsächlich an die Herrschaftskanzlei in Lundenburg zurückgeschickt. Die ehe- 
malige Braut kam in ihre alte Jurisdiktion zurück. Dieser Fall ist in mehrfacher Hinsicht wichtig: er belegt das vorschriftmäßige Handeln der Herrschaftsverwaltung; was noch interessanter erscheint: der Entlaßschein wurde am 15. Februar 1849 retourniert, obwohl inzwischen am 7. September 1848 die Untertänigkeit aufgehoben wurde. Die alten bürokratischen Wege scheinen nicht gleich verlassen worden zu sein. Da es sich um eine jüdische Ehe handelte, wurde natürlich erwähnt, dass der Bräutigam Familiant ist. Die religiösen Konsequenzen, die die nicht realisierte Hochzeit bestimmt hatte, waren für die Herrschaftsverwaltung nicht wichtig.

\section{Neue Richtlinien für die herrschaftliche Jurisdiktion: Der Staat als Schutzherr von Untertanen}

Die Beschränkung der obrigkeitlichen Macht auf dem Gebiet des Rechtswesens wurde bereits im Mai 1781 deutlich. „Die Bestrafung der Untertanen mit Stockstreichen von den obrigkeitlichen Beamten und Schaffersleuten wird allgemein verboten, und dergleichen Züchtigungen sind nicht anders, als nach vorhergehender förmlichen Untersuchung wider strafbare Untertanen zu verhängen."16 Diese Verordnung zeigt die deutliche Tendenz der Reform der Gesetzgebung, wenn es auch beim heutigen Stand der Forschung eine breitere Analyse noch nicht möglich ist. Deutlich ist die bisher nicht erörterte Tendenz, die herrschaftliche Jurisdiktion zwar nicht direkt zu beschränken, sondern sie der Kontrolle von Kreisämtern zu unterstellen.

Dies war ein Bestandteil der vorbereiteten Reform des Gerichtswesens, die zum Ziel die Vereinheitlichung der Jurisdiktion hatte. Am 1. Januar 1782 wurde die Gleichheit aller österreichischen Staatsbürger vor dem Gesetz proklamiert. Das Vorrecht des Adels bloß unter Ehrenwort auszusagen wurde aufgehoben, Adelige mussten wie alle anderen Staatsbürger unter Schwur aussagen. Ab 1. Mai 1783 eröffneten ihre Tätigkeit das Appelationsgericht und das Mährisch-schlesische Landesgericht in Brünn, einen Monat später das böhmische Pendant beider Gerichte in Prag. Die neue Struktur wurde durch die Kreisgerichte ergänzt, die alten Munizipal- und Herrschaftsgerichte wurden beibehalten, die Tätigkeit der Letztgenannten wurde jedoch den Kreisämtern unterstellt.

"Jeder Untertan soll den landesfürstlichen Befehlen sowohl als den Anordnungen der Grundobrigkeit und ihren Beamten gehorchen und unterwürfig sein. "17 Der erste Paragraf des Hofdekrets vom 30. Mai 1781 scheint bloß das „alte Gesicht“ des Absolutismus zu zeigen. Besonders die empfohlene Methode bei Ungehorsam, Störung der öffentlichen Ruhe, gewalttätiger Widersetzung und Gewalt gegen obrigkeitlichen Beamten, nämlich peinliche Aburteilung, erscheinen als nicht besonders modern. Dies ist bloß eine Seite der neuen Stellungnahme von aufgeklärten Regierungskreisen.

Die andere ist die Schaffung der Kontrolle der obrigkeitlichen Gerichtsbarkeit durch politische Ämter. Obrigkeiten waren seit dem Mai 1781 verbunden, ein Verhör- und

16 Hofdekret vom 30. Mai 1781. Joseph II. - Gesetze. Band 1 (1785), S. 51.

$17 \S 1$, Untertanbeschwerdenabhilfe, Hofdekret vom 30. Mai 1781, Joseph II. - Gesetze. Band 1 (1785), S. 47. 
Strafprotokoll zu führen, das dem Kreisamt alle acht Tage zur Kontrolle vorzulegen war. Der Beschuldigte hatte das Recht, sein Vergehen selbst zu erklären. Das Verhör selbst sollte im Beisein des Richters oder anderer „Nachbarn“ verlaufen, so dass niemand bloß auf der Grundlage einer einseitigen Beschuldigung verurteilt werden sollte. Es wird eine Tendenz nach Vereinheitlichung der obrigkeitlichen Jurisdiktion deutlich, nämlich die Festlegung von Strafen: „anständiger, und der Gesundheit nicht nachtheiliger Arrest, allenfalls bei Wasser und Brode, die Strafabeit, die Verschärfung des Arrestes und Strafarbeit mit Anlegung der Fußeisen und die Abstiftung von Haus und Hofe."18

Körperliche Strafen von herrschaftlichen Beamten waren seitdem verboten. Strafen, die über acht Tage hinausgehen oder die Abstiftung vom Haus und Hof sollten vor dem Vollzug vom Kreisamt bestätigt werden. Herrschaftliche Strafprotokolle wurden alle acht Tage zur Kontrolle zugesendet. Dieser wichtige Mechanismus, der ohne finanziellen Aufwand war, ermöglichte die Kontrolle der obrigkeitlichen Strafen durch das Kreisamt und den Vergleich im Rahmen des Landes. Es war also möglich, besonders die großen Dominien zu vergleichen und eventuelle zahlreiche Strafen durch lokale strengere Verhältnisse oder durch die Verwaltung der Herrschaft zu begründen; z. B. ob verhängte Strafen in den Liechtensteinischen Dominien denen in anderen entsprechen oder nicht.

Die Forschung der letzten fünfzig Jahre erörtert das Zurückziehen des Strafprozesses in der Aufklärungszeit aus der Öffentlichkeit (Michel Foucault u. a.). Dies korrespondiert nicht mit dem Hofdekret vom 28. Oktober 1781, nach dem die an einem entfernten Ort vollzogenen Strafen „gegen widerspenstige und unruhige Untertanen keinen wirksamen Eindruck zurücklassen“. ${ }^{19}$ Es wird empfohlen, die Strafe „an Ort und Stelle“ zu vollziehen, die über den Parteien stehende Stellung der Kreisämter wird betont, da die Bestrafung nicht am Kreisamt stattfinden soll.

Der Staat erscheint ganz deutlich als Schutzherr von Untertanen, seine verlängerte Hand - das Kreisamt - ist befugt, die obrigkeitlichen Beamten zu kontrollieren und sogar zu strafen. Falls Obrigkeiten und Beamten die Untertanen „wider Recht und Billigkeit drücken oder mißhandeln“, kann das Kreisamt „eine angemessene Strafe“ verordnen: von 10 bis 50 Gulden; die höchste Geldbuße könnte in behandelter Zeit ein vierteljähriges Gehalt eines gut bezahlten obrigkeitlichen Beamten ausmachen. Neu ist auch Festlegung einer Kerkerstrafe für Beamten, die zum dritten Mal ihre Kompetenzen gegenüber Untertanen übertreten sollten. ${ }^{20}$ Der Schutzherr von Untertanen wurde die Person des Kreishauptmanns: „[...] Wo und immer eine solche Bedrückung entdeckt wird, hat der Kreishauptmann den wider ein klares Gesetz verletzten Untertan ohne Verzug wider alle Zudringlichkeit zu schützen, und schadlos zu halten. "21 Gerade dieser Punkt ist äußerst schwierig, könnte es sich doch um einen Kompetenzkonflikt handeln, falls der Kreis-

18 Die Strafe der Abstiftung vom Haus und Hof wurde jedoch als die äußerste den Dominien empfohlen. Der wirtschaftliche Aspekt des Staates tritt hier sehr deutlich in den Vordergrund. Hofdekret vom 30. Mai 1781. Joseph II. - Gesetze. Band 1 (1785), S. 51.

19 Hofdekret vom 28. Oktober 1781, publiziert als Instruktion für die Kreisämter, Joseph II. - Gesetze. Band 1 (1785), S. 53-57.

20 Ebenda, S. 56

21 Ebenda, S. 55. 
hauptmann zugleich ein Großgrundbesitzer war. Bei der nicht existierenden Forschung $\mathrm{zu}$ diesem Thema kann man dies bloß als eine theoretische Möglichkeit in Betracht ziehen, jedoch muss man auch erwägen, dass vom Standpunkt des Herrschers keine andere Lösung möglich war, da die meisten Ämter durch privilegierte Männer aus Adel und Klerus besetzt waren. Joseph II. hatte also keine andere Möglichkeit zum Schutz von Untertanen, als die angestellten Repräsentanten des Staates.

In der imaginären Hierarchie neuer Rechte von (immer noch) Leibeigenen gehen wir noch weiter. Der Staat bietet den (noch) Leibeigenen die Möglichkeit, sich auf dem Rechtswege gegen herrschaftliche Strafen, bzw. gegen die gerichtliche dominikale Praxis zu beschweren. Der beschuldigte (noch) Leibeigene hatte das Recht unentgeltlich eine Abschrift seines Strafprotokolls zu erhalten, um sich eventuell beschweren zu können. Es ist jedoch in den Gesetzen die Angst vor den großen Bauernaufständen der siebziger Jahre des 18. Jahrhunderts zu spüren. Die Untertanen sollen nicht im Falle eventuellen Unrechts oder Missbrauchs „eigene Richter sein“, sondern die Möglichkeit haben, sich „bei Bekränkung nach der oberen Ordnung (zu) beschweren“, jedoch „inzwischen alle Aufträge umso gewisser vollziehen“. ${ }^{22}$

War der ehemalige Leibeigene wirklich frei? Zum Umzug oder natürlich zur Aussiedlung musste er nach wie vor eine Bewilligung seiner Obrigkeit haben. Was hat sich eigentlich geändert? Besonders interessant ist in diesem Zusammenhang die Dienstbotenordnung von 1782,23 die die rechtliche Stellung von untersten Schichten der Bevölkerung in den neuen Verhältnissen nach der Aufhebung der Leibeigenschaft regelte. Die Aufhebung der Leibeigenschaft (am 1. November 1781) war die erste unausweichliche Stufe zur Freiheit der Person und damit auch zum Bürger. Dem ehemaligen Leibeigenen konnte jetzt die freie Mobilität und das Recht auf persönliche Wahl (Heirat, Bildung) gestattet werden, aber der Staat wollte die volle Kontrolle behalten, um Leute zählen, besteuern und vor allem wieder auffindbar machen zu können, was besonders für das Militär wichtig war, da eben besitzlose Bevölkerungsschichten die meiste Anzahl an Soldaten lieferten. Gegen eine Arbeitsmobilität, die bei armen Menschen am höchsten lag, bestanden sicher keine Einwände, da es der staatlichen Intention der wirtschaftlichen Entwicklung der Monarchie entsprach und in voller Übereinstimmung der regierenden Kreise war, unter denen sich zahlreiche Industrielle befanden. Der umfassende Kontrollmechanismus der Leibeigenschaft musste durch andere Mittel ersetzt werden, in erster Linie durch Kontrolle und Evidenz von Schichten, die nicht an den Besitz gebunden waren. In den im September 1782 eingeführten Dienstbüchern wurde als Grundeinheit die Gemeinde, und nicht die Herrschaft, wie dies bei Pässen, Entlaßscheinen und anderen Personalurkunden war, definiert, was als einer der ersten Züge des neuen Systemdenkens in der Zuordnung von Menschen angesehen werden könnte. Die Gemeinde wird allmählich zum Zentrum der Ordnung, Evidenz, Sicherheit und Fürsorge, was wesentlich später - 1848 - in der Aufhebung der Untertänigkeit und der Übergabe der Verwaltung an die Gemeinden mündete.

22 Hofdekret vom 30. Mai 1781. Joseph II. - Gesetze. Band 1 (1785), S. 49.

23 Die Gesindordnung für das Landgesind in Böhmen, Mähren und Schlesien vom 8. Februar 1782, Joseph II. - Gesetze. Bd. 1 (1785), S. 84. 
Der Übergang vom Leibeigenen zum Bürger scheint sich langsam und kontinuierlich zu ergeben, höchstwahrscheinlich aus Angst, Kontrolle zu verlieren und nicht mehr die Möglichkeit zu haben, alte Mechanismen anwenden zu dürfen: „Würde jemand einen Dienstboten durch Geschenke oder Verheissungen mehreres Lohnes zur Verlassung des Dienstes bereden: so ist derselbe, und eben die Unterhändler dessen mit Geld oder Arrest zu bestrafen, und die Dienstboten zu züchtigen. " ${ }^{24}$ Die Androhung von strengen (auch körperlichen) Strafen zeigt die Stellungnahme der Machtinhaber und deren Furcht, dass die alte Hierarchie auf einmal zusammenstürzen könnte. Warum aber so spät, erst fast ein Jahr nach der Aufhebung der Leibeigenschaft? War nicht etwa der Kaiser zu Zugeständnissen seitens des Adels gezwungen? Der Anspruch des „neuen Bürgers“ - (des ehemaligen Leibeigenen) auf Schutz durch den Staat gegen die unmenschliche Behandlung seitens des Dienstherren war zwar eher theoretisch, da sich der Betroffene außer der „Gefahr an seinen Gliedern oder Leben Schaden zu leiden" nicht von seinem Arbeitsplatz entfernen durfte, ${ }^{25}$ aber er bot bereits die Möglichkeit, seinen Arbeitsgeber für eine schlechte Behandlung anzuzeigen, war also eines der neuen bürgerlichen Rechte. Die eben nur auf der theoretischen Ebene existierenden bürgerlichen Rechte wurden von den Zeitgenossen scharf kritisiert. „Noch erst vor zwölf Jahren mußte man im orthodoxen Polen ein Gesetz machen, daß der christliche Edelmann den christlichen Bauern nicht bloß aus Laune totschlagen soll." 26

\section{Schlussfolgerungen}

Eine prinzipielle Frage drängte sich der Autorin auf: Was zeigen eigentlich die erhaltenen Quellen? Bedeutet die Tatsache, dass bloß Untertanen, die vorschriftlich mit Entlaßscheinen umgezogen waren, gefunden werden, dass es anders (also nicht legal) nicht geschehen konnte? Sehen wir durch die erhaltenen Quellen bloß die Spitze des Eisbergs? Vielleicht ist meine These allzu positiv, aber ich glaube an das richtige Bild, das uns die Archivquellen vermitteln. Wenigstens bei österreichischen Staatsbürgern männlichen Geschlechts. Der Entlaßschein und die Aufnahmeurkunde bedeuteten die Legalität des Inhabers in der neuen Herrschaft, er wurde danach weiter als einheimisch konskribiert und hatte dementsprechend Recht auf die Armenfürsorge im Falle des Alters, der Krankheit, des Todes des Ernährers oder einer vorübergehenden schlechten materiellen Lage. Die Entlasßscheine konnten vermutlich als Reisezeugnis und Identifikationsurkunde, also die Vorstufe des Personalausweises, dienen. Dies war unter der armen Bevölkerung bekannt. Jeder Mann, der ohne Identifikationsurkunde durch Kontrollorgane angehalten wurde, konnte zum Militär eingezogen werden. Er konnte sich nicht rechtlich wehren, dies war ganz im Einvernehmen mit den damals gültigen Gesetzen. Militärangehörige warben sehr gerne und häufig fremde Männer, da sie mit ihnen

$24 \S 24$, Patent vom 30. September 1782, Joseph II. - Gesetze. Bd. 1 (1785), S. 92.

$25 \S 61$, ebenda, S. 98.

26 Pezzl, Johann: Marokkanische Briefe. Frankfurt - Leipzig 1785, S. 40. 
ihr Werbekontingent füllen und demnächst dann weniger „ihrer“ Männer (d. h. Männer aus ihrem Werbbezirk) werben mussten. Hier bot sich ein breiteres Feld für (von mir vermutete Korruption) der zuständigen Werbesubjekte; es bestanden also von der Seite der Untertanen prinzipielle Gründe, um nach Gesetzen und Vorschriften zu handeln.

Wie war es mit Dominien? Würden Herrschaften fremde Untertanen aufnehmen? Wäre es doch nicht in ihrem Sinn, mehr arbeitende Hände zu haben und dadurch wirtschaftlich stärker zu sein? Natürlich ja, aber es war möglich zu überprüfen, ob sich die Herrschaft nicht solidarisch verhält, da die Untertanen bei der nächsten Revision der Konskription gesucht wurden. In diesem Zusammenhang ist die wachsende Macht des Staates nicht zu unterschätzen, da der staatliche Apparat so ein Verhalten eines Dominiums nicht dulden würde. Man kann natürlich noch „die Standesehre“ in Betracht ziehen, die ein gegen andere adelige Besitzer gehendes Verhalten nicht ermöglicht hätte.

Anders war es natürlich mit Menschen, die nicht österreichische Staatsbürger waren. Alle Staaten warben geheim im Ausland, um vor allem qualifizierte Kräfte oder Leute, die bereit waren, ungastliche oder gefährliche Gegenden zu besiedeln, zu bekommen. Den Wert solcher Kräfte zeigt die Höhe der Belohnung für die Festnahme eines fremden „Emissärs“, der österreichische Staatsbürger zur Emigration lockte: 100 Gulden. ${ }^{27}$ So ein Emissär in österreichischen Diensten war Ingenieur Freiherr von Nolde, der durch Mähren nach Galizien „im kaiserlichen Befehl“ reiste. Er wurde mit zahlreichen Privilegien ausgestattet, da er „preußische Immigranten und Webergesellen“ begleitete. Seine Reisedokumente sicherten den freien Weg nicht nur für ihn, sondern für alle mit ihm reisenden Personen „in allen Städten Mährens und Schlesiens“, zuständige Behörden wurden aufgefordert, ihm Hilfe zu leisten. ${ }^{28}$

Ebenso ein beauftragter „Emissär“ war ein gewisser Mickerte, der nach Limburg abgeschickt worden war, um Spezialisten für die Textilindustrie zu werben. Er gewann 23 Niederländer, davon drei Frauen, die er im Mai 1798 nach Frankfurt begleitete (tatsächlich kamen 26 Personen). Er handelte wahrscheinlich im Auftrag der Inhaber der Textilfabrik im mährischen Ort Namiest (Náměšṫ), die auch die Reisedokumente erledigt zu haben schienen. Aus der Korrespondenz zwischen dem geschäftsführenden Mitbesitzer der Manufaktur Johann von Puthon ${ }^{29}$ und dem österreichischen Polizeiminister Johann von Pergen ${ }^{30}$ geht hervor, dass die Einwanderung mit der Zustimmung der höchsten

27 Hofdekret vom 16. März 1787, Joseph II. - Gesetze. Bd. 13 (1789), S. 462-463.

28 Instruktionen für Ingenieur von Nolde vom 29. März 1784, MLA Brünn, Mährisch-schlesisches Landesgubernium P 25 B, Karton 1427; Brief an Repräsentation und Kammer vom 18. März 1784, der die reisende Gruppe vorausging, ebenda.

29 Johann Baptist Edler von Puthon (1744-1816), Reichsfreiherr, österreichischer Industrielle und Großhändler, betrieb zusammen mit seinen Söhnen Johann Baptist (1776-1839) und Karl (1780-1863) mehrere Textilunternehmen, u. a. die Katunmanufaktur in Sassin (Oberungarn, heute die Slowakei), die Baumwollmaschinenspinnerei in Teesdorf (Niederösterreich), die Cottonfabrik in Friedau (Niederösterreich); seine Feintuchfabrik in Náměšt (Mähren) gehörte zu besten spezialisierten Betrieben Österreichs. Außerdem war er als Besitzer des geerbten Großhandels G. J. Schuller \& Comp. als Großhändler tätig. MLA Brünn, B 95, Karton 244, Sign. 55; Neue deutsche Biographie. Bd. 21. Berlin 2003, S. 18-19; Bd. 18. Berlin 1997, S. 47; Österreichisches Biographisches Lexikon 1815-1950. Bd. 8. Wien 1982, S. 346.

30 Johann Anton Graf von Pergen (1752-1814), österreichischer Diplomat und Staatsmann, versuchte als Polizeiminister Josephs II. gegen Widerstände der Hofkanzlei und Landesregierungen das Polizeiwesen 
Stellen geschah. Die Polizeihofstelle wandte sich in Person des Stellvertreters des Polizeiministers Joseph von Saurau, noch bevor die Einwanderer österreichischen Boden betraten, an beide Landesgouverneure, durch deren Länder Immigranten reisen sollten, an den böhmischen und den mährisch-schlesischen, um sie nicht nur zu verständigen, sondern diesen auch höflich aufzutragen, den Niederländern die Fortsetzung der Reise nach Namiest in Mähren, wo die Fabrik ihrer unumgänglich bedarf, zu gestatten. "31 Der Prozess geschah an der Grenze zur Legalität, da sich voraussetzen lässt, dass Niederländer keine Pässe, Entlaßscheine oder andere Dokumente hatten. Durch die Vermittlung eines Handelshauses, das dies bestimmt nicht ohne Gegenleistung machte, bekamen sie in Frankfurt am Main Reisepässe. Österreichischen Boden betraten sie also ganz legal. Sie wurden nach der Ankunft in Namiest ordentlich angemeldet. Ihre Ansiedlung erleichterte sicher den Umstand, dass der Großgrundbesitzer Heinrich von Haugwitz auch der Mitbesitzer der Manufaktur war. Die Quellen belegen, dass es nicht um einen einmaligen Prozess ging. Johann Baptist von Puthon kündigte noch im selben Jahr 1798 die Ankunft weiterer 14 bis 16 Tuchmacher an, die unter der Leitung eines gewissen Delhaes kommen sollten. Außerdem erwähnte er, dass bereits einige Niederländer in Namiest seien. Ausländische Untertanen wurden hier sehr gerne angenommen und ihre weitere Existenz in Österreich war durchaus legal.

Qualifizierte österreichische Staatsbürger durften übersiedeln, aber nicht aus Österreich auswandern. Im sog. Emigrationspatent vom Jahre 1784 werden bloß „Glasarbeiter aus Böhmen und Eisenarbeiter aus der Steiermark “ genannt, ${ }^{32}$ aber das Postulat wurde auch auf andere Professionen angewendet, z. B. auf Spezialisten in der Textilbranche. Handwerker (nicht Gesellen auf der obligaten Walz) bekamen deshalb von ihren Obrigkeiten schwieriger die Reisezeugnisse. Herrschaftsämter wurden deswegen angewiesen „sehr vorzüglich behutsam zu sein, damit durch unvorsichtige Ertheilung der Pässe nicht vielleicht geschickten Künstlern oder Handwerkern, welche nach dem Verhältnisse der Fabriken ieder Provinz die Nothwendigkeiten sind, die Gelegenheit aus dem Lande zu kommen erleichtert". Dominien und Unternehmern wurde auferlegt, „wenn sie von einer auswärtigen Anlockung etwas erfahren, der Landesstelle davon unverzüglich Nachricht zu geben ". 33

War die Aufhebung der Leibeigenschaft eine so krasse Schwelle, wie die Geschichtsbücher darstellen? Manche Untertanen wussten ihre richtige Obrigkeit nicht, und trotzdem lebten sie seit Jahren ungestört. So ging es einer gewissen Elisabeth Gießhuberin aus Vierhöf (Čtyři Dvory), Herrschaft Budweis (České Budějovice), die nach Wien übersiedelte, ohne die obrigkeitliche Bewilligung geholt zu haben. Außerhalb ihrer Herr-

zu organisieren. Unter Leopold II. war er pensioniert, konnte jedoch unter der Regierung Franz II. in sein Amt zurückkehren. Pergen schuf, zusammen mit seinem Stellvertreter Joseph von Saurau und Franz Anton von Beer, das System der österreichischen Geheimpolizei und bereitete damit den Weg für die innere Politik des Vormärzlichen Österreichs. Neue Deutsche Biographie, Bd. 20. Berlin 2001, S. 185-186; MZA Brno, B 95, Karton 243 und 244, Sign. 55.

31 Franz Joseph von Saurau an den böhmischen Landesgouverneur, MLA Brünn, B 95, Karton 244, Sign. 55 , Folio 17.

32 „Emigrationspatent“ vom 10. August 1784, Joseph II. - Gesetze. Bd. 6 (1786), S. 279 ff.

33 Ebenda, S. 286-287. 
schaft heiratete sie, lebte in Rannersdorf (Niederösterreich) und Wien und zog drei Kinder groß. Nach dreißig Jahren kehrte sie in ihre Jurisdiktion zurück, um das Erbe nach ihrer Mutter zu bekommen. Ihr Gesuch um das Erbe wurde abgelehnt und Elisabeth Gießhuberin wurde wegen ihrer Flucht von der Herrschaft und der nicht bewilligten Eheschließung verhaftet. Im Kerker im Februar 1766 im von ihr eingeschätzten Alter von 53 Jahren suchte sie nachträglich um die Entlassung aus der Jurisdiktion an. Beim Verhör beantwortete sie die Frage, wie es möglich gewesen sei, dass sie ohne Bewilligung geheiratet habe, folgendermaßen: „Ich glaube, er (ihr Ehemann) wird ein Unterthann seyn von der Stadt [...] dann habe ich auch wirklich nicht gewußt, und nicht verstanden, ob ich eine oder keine Untertannin bin. "34 Ihrem Gesuch um die Entlassung aus der Jurisdiktion wurde durch den Budweiser Stadtrat mit Rücksicht auf ihr Alter und den kranken Ehemann stattgegeben. Ihr Erbe fiel nach dem Recht der Stadt zu.

Der obenerwähnte Joseph Kutschera wusste seine Jurisdiktion auch nicht, bzw. lebte seit Jahren im Glauben, dass er Lundenburger Untertan sei. Erst bei seinem Gesuch um die Entlassung wurde festgestellt, dass er eigentlich gar nicht um die Aufnahme in die neue Feldsberger Jurisdiktion bitten müsse, sondern dass er die ganze Zeit deren Untertan war. Beide Akteure waren sich keiner Schuld bewusst. Bei Joseph Kutschera konnte die Tatsache maßgebend sein, dass er in Lundenburg geboren wurde. Den Geburtsort hielt Josef Kutschera wahrscheinlich für sein politisches Domizil und gab ihn falsch bei der Feldsberger Herrschaft an. Seine Mutter war zur Zeit der Antragstellung bereits verstorben, die ihm die Auskunft hätte geben können. Elisabeth Gießhuberin wurde im Alter von sieben Jahren von ihrem Vater aus dem Haus vertrieben und verdiente sich dann mit Kinderbetreuung und später als Dienstmagd ihr Brot. Ihr fehlte das Bewusstsein ihres Zuhause und ihrer Obrigkeit. Beide Fälle trennt eine lange Zeit, E. Gießhuberin wurde im Februar 1766 festgenommen, also vor der Aufhebung der Leibeigenschaft, J. Kutschera reichte sein Gesuch im Januar 1847 an, lange nach der Aufhebung der Leibeigenschaft. Beide Fälle sind sehr ähnlich, obwohl dazwischen die Leibeigenschaftsaufhebung liegt. Wurde die Leibeigenschaft, bzw. die Untertänigkeit überhaupt wahrgenommen? Beide Menschen handelten als freie Personen.

Stellte die Aufhebung der Leibeigenschaft wirklich einen so wichtigen Schritt zur modernen Gesellschaft? Unbestritten ja, aber die Verwirklichung lag nicht bloß in der Aufhebung wichtiger Kontrollmechanismen, die überdies durch andere Kontrollmittel ersetzt wurden, sondern eher im allmählichen Umbau des Rechtsystems. Die dominikale Jurisdiktion wurde sehr beschränkt und zum Teil der Kontrolle von Kreisamt unterstellt, was eben zu den neuen, wenn auch nicht besonders bekannten Merkmalen der Zeit der Leibeigenschaftsaufhebung gehört. Ein neues Merkmal brachte diese Zeit doch: das Recht für ehemalige Leibeigene, sich gegen die herrschaftliche Gewalt und eventuell gegen den Missbrauch gesetzlich wehren zu können.

34 Grulich, Josef: Migrace českobudějovického obyvatelstva ve světle propouštěcích listů (1756-1770) [Die Migration von Budweiser Bevölkerung im Licht der Entlaßscheine (1756-1770)]. In: Profesoru Lumíru Dokoupilovi k sedmdesátinám, [Professor Lumír Dokoupil zum siebzigsten Geburtstag]. Historie - Historica 219, Ostrava 2005, S. 55-71, hier S. 69. 


\section{Zusammenfassung}

\section{Svobodní lidé? Svoboda pohybu bývalých nevolníků v Rakousku 1781-1848 (na př́ikladu lichtenštejnských dominií)}

Př́íspěvek se snaží nalézt odpověd' na otázku, nakolik se změnilo postavení nevolníků po zrušení nevolnictví v Rakousku. Byli skutečně osobně svobodní, jak je dlouhodobě tradováno v syntézách a učebnicích? Na př́kladu sondy do dvou lichtenštejnských panství, jihomoravského dominia Břeclav/Lundenburg a dolnorakouského panství Wilfersdorf byl analyzován faktor osobní svobody pohybu po zrušení nevolnictví. Mohli se bývalí nevolníci skutečně volně stěhovat na jiná panství či do ciziny? Úzce vymezená analýza ukazuje, že nikoliv, a to až do konce doby předbřeznové, tedy až do zrušení poddanství. Toto zjištění je v rozporu s tradovaným výkladem. Zrušení nevolnictví bylo bezesporu krokem k moderní společnosti, avšak její realizace nespočívala v odstranění kontrolních mechanismů, jež byly ostatně nahrazeny jinými, nýbrž v postupné přestavbě právního systému. Nepřiliš známým novým faktorem doby je omezení dominikální jurisdikce, jež byla postavena pod kontrolu krajských úřadů. Nový jev však reformní období přece přineslo: zákonně ukotvené právo bývalých nevolníků bránit se vrchnostenské moci. 